\title{
Levy Mutated Artificial Bee Colony Algorithm for Global Optimization
}

\author{
Anguluri Rajasekhar \\ Department of Electrical and Electronics Engineering \\ National Institute of Technology-Warangal \\ Andhra Pradesh, India \\ Email: rajasekhar.anguluri@ieee.org
}

\author{
Ajith Abraham \\ Faculty of Computer Science and Electrical Engineering, \\ VSB - Technical University of Ostrava, Czech Republic \\ Machine Intelligence Research Labs (MIR Labs), USA \\ Email: ajith.abraham@ieee.org
}

\author{
Millie Pant \\ Department of Paper and Pulp Technology \\ Indian Institute of Technology-Roorkee \\ Roorkee 247667, INDIA \\ Email: millifpt@iit.ernet.in
}

\begin{abstract}
This paper proposes an improved version of Artificial Bee Colony (ABC) algorithm with mutation based on Levy Probability Distributions. The Levy distribution has a peculiar property of generating an offspring farther away from its parent which depends on internal parameter $\alpha$ compared to that of Gaussian mutations, this property enables in finding out most optimal solutions to the problems than that of conventional methods. The proposed algorithm is tested on 7 standard benchmark functions and on a set of non-traditional problems suggested in the special session of CEC'2008. Analysis and comparison of results with other state of art optimization algorithms like GA and PSO, shows the superiority of improved mutation, especially on high dimensional problems. This paper finally investigates the performance of proposed algorithm on the frequency-modulated sound wave synthesis problem, a real world problem in the field on communication engineering
\end{abstract}

Keywords-artificial bees; levy probability distributions; global optimization;

\section{INTRODUCTION}

Artificial Bee Colony (ABC) is one of the most recent, swarm intelligence based algorithms employing the foraging behavior of honey bees for solving dynamic numerical optimization problems. ABC was suggested by Karaboga and Basturk for function optimization $[1,2]$ in 2005. Due to its simplicity and robustness it has been successfully applied to various practical optimization problems like Clustering [3]; IIR filter design [4]; extraction of MESFET [5] and so on; However like most of the population based algorithms, ABC also has some non-appreciable drawbacks. As pointed by Zhu and Kwong [6], the structure of $\mathrm{ABC}$ is such that it supports global exploration more in comparison to the exploitation. However, the performance of an algorithm depends on both exploration and exploitation phases for a required feasible solution.

Various modifications have been suggested in literature to improve performance of ABC. Some interesting modifications include virtual bee algorithm (or VBA) in which the bees moves randomly in the phase space and interact by finding odd sources corresponding to the encoded values of the function [7]. Sundareswaran et al. proposed an approach based on natural behavior of honey bees in nectar collection in which the randomly generated worker bees are forced to move in the direction of elite bee [8]. Karaboga et al. analyzed the performance of $\mathrm{ABC}$ based on the parameter tuning [9]. Abraham et al. proposed hybrid $\mathrm{ABC}-\mathrm{DE}$ which combines ABC with Differential Evolution [10].

In this present study an external mutation operator based on Levy distribution is embedded into the structure of basic $A B C$. The performance of the proposed algorithm called L-ABC is validated on standard benchmarks [11] and also on composite functions presented at Congress on Evolutionary Computation 2008 (CEC'2008) [12]. Finally we investigated the performance of proposed scheme on frequency modulated sound wave synthesis problem. Levy probability distribution has an infinite second moment unlike to that of Gaussian distribution. One of the interesting features of Levy Probability distributions [11] is its power law in the tail region. The power law implies that there is no characteristic length scale and this is the mile stone of fractal structure. Further more flexibility in levy distribution is that by adjusting the parameter $\alpha$, with this one can tune the probability density, which in turn yields adjustable variation in the mutations.

The rest of paper is organized as follows. In Section II, we discuss the basic version of ABC. Section III gives a brief overview of levy distribution followed by the proposed Levy mutated ABC (L-ABC). Section IV provides detailed comparison between the $\mathrm{L}-\mathrm{ABC}$ and remaining state of art optimization methods on a test suite of numerical benchmarks. Section V describes an application of the L-ABC to the parameter estimation problem for frequency-modulated sound wave. Finally we end the paper with some conclusions given in Section VI.

\section{ARtificial BeE COLONY Algorithm}

Artificial Bee Colony (ABC) algorithm classifies the foraging artificial bees into three groups; the employed bees, the onlooker bees and the scouts. The first half of the colony 
consists of the employed bees and second half consist of the onlooker bees. A bee that is currently searching for food or exploiting a food source is called an employed bee and a bee waiting in the hive for making decision to choose a food source is called an onlooker bee. For every food source, there is only one employed bee. The employed bee of abandoned food source becomes a Scout. In ABC algorithm each solution to the problem is considered as food source and is represented by a $D$-dimensional real-valued vector, where the fitness of the solution corresponds to the nectar amount of associated food source. Like other swarm based algorithms, ABC is also an iterative process.

The algorithm starts by initializing all the employed bees with randomly generated food sources (solutions). In each generation/iteration every employed bee finds a food source in the neighborhood of its current food source and evaluates its nectar amount i.e., (fitness). In general the position of $i_{t h}$ food source, for a D dimensional search space, is represented as $X_{i}=\left\{x_{i 1}, x_{i 2}, \ldots . x_{i D}\right\}$.After the information is shared by the employed bees; onlooker bees go to the region of food source at $X_{i}$ based on the probability $P_{i}$ defined as

$$
P_{i}=\frac{f i t_{i}}{\sum_{k=1}^{F S} f i t_{k}}
$$

$F S$ is total number of food sources. Fitness value fit $_{i}$ is calculated by using following equation.

$$
\text { fit }_{i}=\frac{1}{1+f\left(X_{i}\right)}
$$

Here $f\left(X_{i}\right)$ is the objective function to be minimized. The onlooker finds its food source in the region $X_{i}$, by making use of following equation.

$$
x_{n e w}=x_{i j}+r^{*}\left(x_{i j}-x_{k j}\right)
$$

Where $k \in(1,2,3, \ldots, F S)$ such that $k \notin i$ and $j \in(1,2,3, \ldots, D)$ are randomly chosen indexes, $r$ is a uniformly distributed random number in the range $[-1,1]$.

If the obtained new fitness value is better than the fitness value achieved so far, than the bee moves to this new food source leaving this old one otherwise it retains the old food source. When all employed bees have completed this process, the information is shared with onlookers. Each of the onlookers selects a food source according to the probability given above. By this scheme good sources are well accommodated with onlookers. Each bee will search for a better food source for a certain number of cycles (limit), and if the fitness value doesn't improve then that particular bee becomes a Scout bee. A food source is initialized to that scout bee randomly and the search process continues. In this approach we used basic version which involve only one scout bee.

\section{L-ABC: LEVY MutATED ARTIFICIAL BEE COLONY ALGORITHM}

According to Karaboga, $\mathrm{ABC}$ has only one control parameter 'limit' apart from the common control parameters of the population-based algorithms such as population size or colony size $(N B)$ and maximum generation number or maximum cycle number $(M C N)$. The basic version of $\mathrm{ABC}$ is very powerful in solving Uni-modal and Multimodal functions of considerable dimensions. However, the convergence rate and quality of solutions deteriorates with the increase in the problem complexity.

As mentioned earlier the basic structure of $\mathrm{ABC}$ favors exploration more in comparison to exploitation as a result some important domain knowledge may get lost during the successive iterations. In order to enhance the exploitation capabilities of $\mathrm{ABC}$, some added mechanism is needed for which we employed Levy mutation (based on Levy probability distribution) to assist $\mathrm{ABC}$ in exploitation of the search space.

\section{A. Levy Probability Distribution}

In 1930s, P. Levy, introduced a distribution which gives an infinite second moment, unlike finite second moment in Gaussian distribution, known as Levy's Probability Distribution [11]. It is also a stable process with an infinite moment which provides a characteristic tail at the end. The distribution is given as follows.

$$
L_{\alpha, \gamma}(y)=\frac{1}{\pi} \int_{0}^{\infty} e^{-\gamma q^{\alpha}} \cos (q y) d q
$$

From Eqn. (4), we see that the distribution is symmetric with respect to $y=0$ and has two parameters $\gamma$ and $\alpha . \gamma$ is the scaling factor satisfying $\gamma>0$ and $\alpha$ should satisfy $0<\alpha<2$. Considering the limits in particular for $\alpha=1$, the integration can be analytically performed where it is equivalent to Cauchy Probability distributions. For limit $\alpha \rightarrow 2$, the distribution move towards Gaussian distribution. The parameter $\alpha$ controls the shape of the distribution in such a way that one can obtain different shapes of probability distribution, especially in the tail region.

The smaller is the parameter $\alpha$ longer is the tail of the distribution. Levy distribution provides an ideal probability distribution for designing mutation operators because of its adjustable parameter. Different $\alpha$ values define probability distributions of various shapes. For example, it can implement both Gaussian and Cauchy distributions by simply changing the parameter $\alpha$. The complete mathematical and theoretical concepts of Levy Probability distribution can be found in [13].

\section{B. Levy Mutated Artificial Bee Colony}

The performance of Artificial Bee colony depends on exploration and exploitation which is done by the scout and employed bees respectively. The main motivation behind mutation is to guide the virtual bees towards the global optimum within less computational time (fast convergence) while maintaining the quality of solutions.

In order to apply Levy mutation we made use of Rechenberg's $1 / 5$ rule [9], which adapts the mutations step size depending on the frequency of successful mutations. This rule states that the ratio of successful mutations to all mutations should be $1 / 5$; hence if the ratio is greater than $1 / 5$ the step size increases, and if the ratio is less $1 / 5$ step size decreases. However, while performing the experiments we observed that the Rechenberg's ratio can be reduced further in 
case of $\mathrm{ABC}$ algorithm therefore in the present study we have taken the ration as $1 / 10$.

A new food source $X_{i}=\left\{x_{i 1}, x_{i 2}, x_{i 3}, \ldots, x_{i D}\right\}$ by L-ABC is generated as follows

$$
x_{n e w}=x_{i, b e s t}+L(\alpha, 0)
$$

Here $L(\alpha, 0)$ stands for random number generated by Levy's distribution. The mutation rule is applied making use of eq. (5).

From our experimentations we observed that $\gamma=1$ and $\alpha=1.5$ are the most suitable parameters in the current study.

\section{Pseudo codes of $\mathrm{ABC}$ and $\mathrm{L}-\mathrm{ABC}$}

\section{(1) Basic Artificial Bee Colony (ABC)}

Step1. Initialize the population of solutions $x_{i j}, i=1,2, \ldots F S, j=1,2, \ldots D$, trial $_{i}=0$

trail $_{i}$ is the non-improvement number

Step2. Evaluate the population

Step3. Cycle $=1$

Step4. REPEAT

\{----Produce new food source population for employed bee----- $\}$

Step5. For $i=1$ to $F S$ do

i. Produce a new food source $v_{i}$ for the employed bee of the food source $x_{i}$ by using (3) (in case of L-ABC use eq. (5) to evaluate the amount of nectar)

ii. Apply a greedy selection process between $v_{i}$ and $x_{i}$ and select the better one

iii. If solution $x_{i}$ doesn't improve trial $_{i}=\operatorname{trial}_{i}+1$, otherwise trial $_{i}=0$;

End for

Step6. Calculate the probability values $P_{i}$ by (4) for the solutions using fitness values.

$\{$---Produce new food source population for onlooker bee--- $\}$

i. $\quad \mathrm{t}=0$;

ii. $\quad \mathrm{i}=1$;

Step7. REPEAT

If rand $<P_{i}$ then

i. Produce a new food source $v_{i}$ for the employed bee of the food source $x_{i}$ by using (3) (in case of L-ABC use eq. (5) to evaluate the amount of nectar)

ii. Apply a greedy selection process between $v_{i}$ and $x_{i}$ then select the better one

iii. If solution $x_{i}$ doesn't improve trial $_{i}=$ trial $_{i}+1$, otherwise trial $_{i}=0 ;$

iv. $\mathrm{t}=\mathrm{t}+1$

\section{End if}

UNTIL (t=FS)

\{--------Determine Scout--------- $\}$

Step8. If $\max ($ trial $)>$ limit then

i. Replace $x_{i}$ with a new randomly produce solution by using following equation $x_{i j}=x_{j}^{\min }+\operatorname{rand}(0,1) *\left(x_{j}^{\max }-x_{j}^{\min }\right)$

End If

ii. Memorize the best solution achieved so far Cycle $=$ Cycle +1 ;

UNTIL (Cycle=Maximum Cycle Number)

\section{(2) Levy Mutated Artificial Bee Colony (L-ABC)}

All the algorithmic steps of L-ABC are same as that of $\mathrm{ABC}$ except that Levy mutation is applied using the modified Rechenberg's rule.

\section{EXPERIMENTS AND RESULTS OVER BENCHMARK FUNCTIONS}

This section presents an extensive comparison among the performances of L-ABC algorithm with basic $\mathrm{ABC}$, real coded GA, and PSO. We considered a test suite of 7-well known benchmark functions of very high complexity and also 3 functions of nontraditional shifted benchmark problems that are suggested in CEC 2008. In Table II dim represents the dimension of the problem. We used 100 dimensions for functions $f_{1}$ to $f_{6}$ while function $f_{7}$ is 2 -D. The rest of the functions belong to the class of nontraditional shifted functions each function having dimension of 500. Table II summarizes the different benchmark functions that are being used in the present study.

\section{A. Parameteric Set up Used for the Alogirthm}

Various parameters employed for the $\mathrm{ABC}$ and also for $\mathrm{L}-\mathrm{ABC}$ algorithms are summarized in Table I. There is not much difference in the parametric set up of both the algorithms except for the scaling parameter. The obtained values of test functions for 30 independent runs are recorded in Table III.

Table I Parameter Settings of ABC and L-ABC

\begin{tabular}{|l|l|}
\hline Parameter & Value \\
\hline No of Bees (NB) & $\begin{array}{l}20 \text { (for 100-D problems), } \\
50 \text { ( for 500-D problems) }\end{array}$ \\
\hline Food Sources (FS) & $\mathrm{NB} / 2$ \\
\hline Employed bees & $50 \%$ of bees \\
\hline Onlooker bees & $50 \%$ of bees \\
\hline Scout bees & 1 \\
\hline Limit & $n_{e}^{*} \mathrm{D}$ \\
\hline Scale Parameter & 1.5 \\
\hline
\end{tabular}

$\mathrm{D}$ - dimension of the problem

\section{B. Empircal Results}

Table III and IVcompares the basic version of ABC and proposed L-ABC on the quality of best soulutions obtained. 
The mean and standard deviation of the best-of-run 30 independent runs are presented in Table III and Table IV for 7 traditional benchmarks and 3 nontraditional shifted functions respectively. The proposed algorithm is also compared with Genetic Algorithm (GA) and Particle Swarm Optimization (PSO) tehniques [14] for the 100-Dimensional functions and on the other hand for 500-Dimensional shifted functions we compared the performance of L-ABC with one of the variants of Differential Evolution called Differential Evolution with Self-adpation and Cooperative Co-evolution (DEwSACc) [15] and with a variant of PSO called Improved Particle Swarm Optimizer. (EPUS-PSO) [16].

Tables V and VI shows the best values obtained by different algorithms used for comparison purpose. The convergence characteristics of functions from $f_{1}$ to $f_{10}$ are provided from Fig 1 to Fig 10. Each graph shows how the objective function value of best in a pupulation changes with increasing number of FEs. A termination criterion of 1,00,000 FEs are used for $f_{1}$ to $f_{7}$ and that of 10,00,000 FE's are used for $f_{8}$ to $f_{10}$. From the results it is very clear that L-ABC out performed all the algorithms with a promising solutions and DEwSACc algorithm remained a strong competitor for the L-ABC and which showed a good optimal solution for $f_{11}$ function but according to overall comparisons and simulations it is clear that L-ABC is superior to that of remaining methods.

Table II Description of the Benchmark Functions

\begin{tabular}{|l|c|c|c|c|}
\hline \multicolumn{1}{|c|}{ Function } & Mathematical Representation & $\begin{array}{c}\text { Dim } \\
\text { (D) }\end{array}$ & $\begin{array}{c}\text { Range of } \\
\text { Search (S) }\end{array}$ & $\begin{array}{c}\text { Theoretical } \\
\text { Optima }\end{array}$ \\
\hline Sphere & $f_{1}(\vec{x})=\sum_{i=1}^{D} x_{i}^{2}$ & 100 & $(-100,100)^{D}$ & $f_{1}(\overrightarrow{0})=0$ \\
\hline Rosenbrock & $f_{2}(\vec{x})=\sum_{i=1}^{D-1}\left[100\left(x_{i+1}-x_{i}^{2}\right)^{2}+\left(x_{i}-1\right)^{2}\right]$ & 100 & $(-100,100)^{D}$ & $f_{2}(\overrightarrow{1})=0$ \\
\hline Rastrigin & $f_{3}(\vec{x})=\sum_{i=1}^{D-1}\left[x_{i}^{2}-10 \cos \left(2 \pi x_{i}\right)+10\right]$ & 100 & $(-5.12,5.12)^{D}$ & $f_{3}(\overrightarrow{0})=0$ \\
\hline Grienwank & $f_{4}(\vec{x})=\frac{1}{4000} \sum_{i=1}^{D} x_{i}^{2}-\prod \cos \left(\frac{x_{i}}{\sqrt{i}}\right)+1$ & 100 & $(-600,600)^{D}$ & $f_{4}(\overrightarrow{0})=0$ \\
\hline Ackley & $f_{5}(\vec{x})=-20 \exp \left(-0.2 \sqrt{\frac{1}{D} \sum_{i=1}^{D} x_{i}^{2}}\right)-$ & 100 & $(-32,32)^{D}$ & $f_{5}(\overrightarrow{0})=0$ \\
\hline $\begin{array}{l}\text { Schwefel } \\
\text { Easom }\end{array}$ & $f_{6}\left(\frac{1}{D} \sum_{i=1}^{D} \cos \left(2 \pi x_{i}\right)\right)+20+e$ & & & \\
\hline $\begin{array}{l}\text { Shifted } \\
\text { Sphere }\end{array}$ & Multi-modal, separable, huge number of local minima & 500 & $(-5,5)^{D}$ & $f_{9}=0$ \\
\hline $\begin{array}{l}\text { Shifted } \\
\text { Rastrigin's }\end{array}$ & Multi-Modal, Separable $n-\sum_{i=1}^{n} x_{i} \sin \left(\sqrt{\left|x_{i}\right|}\right)$ & 100 & $(-500,500)^{D}$ & $f_{6}(\overrightarrow{420.97})=0$ \\
\hline $\begin{array}{l}\text { Shifted } \\
\text { Ackley's }\end{array}$ & Uni-modal, separable, scalable $\left(x_{1}\right) \cos \left(x_{2}\right)$ exp $\left[-\left(x_{1}-\pi\right)^{2}-\left(x_{2}-\pi\right)^{2}\right]$ & 2 & $(-2,2)$ & $f_{7}(\pi, \pi)=0$ \\
\hline
\end{tabular}


Table III Comparison of ABC and L-ABC in terms of Error, Standard Deviation and Fitness on traditional benchmarks

\begin{tabular}{|c|c|c|c|c|l|l|}
\hline \multirow{2}{*}{ Fun. } & \multicolumn{2}{|c|}{ Fitness } & \multicolumn{2}{c|}{ Error } & \multicolumn{2}{c|}{ Standard Deviation } \\
\cline { 2 - 7 } & ABC & L-ABC & ABC & L-ABC & ABC & L-ABC \\
\hline$f_{1}$ & $7.8484 \mathrm{E}-007$ & $\mathbf{2 . 6 5 2 5 E - 0 1 5}$ & $4.16757 \mathrm{E}-006$ & $\mathbf{4 . 3 2 1 3 9 E - 0 1 5}$ & $4.55706 \mathrm{E}-006$ & $\mathbf{1 . 9 2 7 9 3 E - 0 1 5}$ \\
\hline$f_{2}$ & $1.31556 \mathrm{E}+002$ & $\mathbf{1 . 1 3 6 0 2 E}+00$ & $2.36941 \mathrm{E}+002$ & $\mathbf{2 . 1 5 4 2 3 E + 0 1}$ & $1.11458 \mathrm{E}+002$ & $\mathbf{2 . 3 2 3 1 4 E}+\mathbf{0 1}$ \\
\hline$f_{3}$ & $8.74222 \mathrm{E}+00$ & $\mathbf{4 . 9 4 6 5 1 4 E - 1 0}$ & $1.56381 \mathrm{E}+001$ & $\mathbf{1 . 0 3 0 9 1 E}+00$ & $3.23844 \mathrm{E}+00$ & $9.72414 \mathrm{E}-001$ \\
\hline$f_{4}$ & $4.046268 \mathrm{E}-006$ & $\mathbf{1 . 2 2 1 2 4 E - 0 1 5}$ & $5.03246 \mathrm{E}-003$ & $\mathbf{4 . 9 6 6 4 9 E - 0 0 4}$ & $9.15662 \mathrm{E}-003$ & $\mathbf{2 . 4 7 1 4 1 E - 0 0 3}$ \\
\hline$f_{5}$ & $8.094955 \mathrm{E}-003$ & $\mathbf{2 . 4 1 4 6 5 E - 0 0 8}$ & $2.96985 \mathrm{E}-002$ & $\mathbf{1 . 0 9 4 1 1 E - 0 0 7}$ & $2.64681 \mathrm{E}-002$ & $\mathbf{1 . 4 0 8 4 3 E - 0 0 7}$ \\
\hline$f_{6}$ & $2.167262 \mathrm{E}+003$ & $\mathbf{6 . 0 4 4 1 7 E}+002$ & $3.19633 \mathrm{E}+003$ & $\mathbf{1 . 1 9 8 6 6 E}+\mathbf{0 0 3}$ & $3.79350 \mathrm{E}+002$ & $\mathbf{3 . 2 8 3 0 9 E}+\mathbf{0 0 2}$ \\
\hline$f_{7}$ & $\mathbf{0}$ & $\mathbf{0}$ & $8.65942 \mathrm{E}-002$ & $\mathbf{7 . 4 0 1 4 9 E - 0 1 8}$ & $4.64696 \mathrm{E}-004$ & $\mathbf{2 . 8 1 6 7 3 E - 0 1 7}$ \\
\hline
\end{tabular}

Table IV Comparison of ABC and L-ABC in terms of Error, Standard Deviation and Fitness on shifted benchmarks

\begin{tabular}{|c|c|c|c|c|c|c|}
\hline \multirow{2}{*}{ Fun. } & \multicolumn{2}{|c|}{ Fitness } & \multicolumn{2}{c|}{ Error } & \multicolumn{2}{c|}{ Standard Deviation } \\
\cline { 2 - 7 } & ABC & L-ABC & ABC & L-ABC & ABC & L-ABC \\
\hline$f_{8}$ & $5.6776 \mathrm{E}-007$ & $\mathbf{6 . 8 1 5 7 E - 0 1 3}$ & $9.3558 \mathrm{E}-006$ & $\mathbf{7 . 7 7 0 2 9 E - 0 1 2}$ & $1.87417 \mathrm{E}-005$ & $\mathbf{9 . 4 2 6 0 5 - 0 1 2}$ \\
\hline$f_{9}$ & $7.73042 \mathrm{E}+01$ & $\mathbf{6 . 9 9 1 4 E + 0 0}$ & $1.10386 \mathrm{E}+02$ & $\mathbf{1 . 4 1 5 8 3 E}+\mathbf{0 1}$ & $1.20619 \mathrm{E}+01$ & $\mathbf{4 . 0 2 3 0 3 E}+\mathbf{0 0}$ \\
\hline$f_{10}$ & $2.94500 \mathrm{E}-01$ & $\mathbf{6 . 5 5 7 4 E - 0 0 6}$ & $5.85487 \mathrm{E}-01$ & $\mathbf{2 . 2 5 7 9 3 E - 0 0 5}$ & $1.58059 \mathrm{E}-01$ & $\mathbf{1 . 2 1 1 8 0 4 E - 0 0 5}$ \\
\hline
\end{tabular}

Table V Comparison of GA, PSO, ABC and L-ABC in terms of fitness value over traditional benchmark functions

\begin{tabular}{|l|l|l|l|l|}
\hline \multicolumn{1}{r|}{ Algorithm } & GA & $\underline{\text { PSO }}$ & $\underline{\text { ABC }}$ & $\underline{\text { L-ABC }}$ \\
\hline Sphere & & & & \\
\hline Rosenbrock & $5.650 \mathrm{E}+01$ & $2.2121 \mathrm{E}+02$ & $7.8484 \mathrm{E}-007$ & $\mathbf{2 . 6 5 2 5 E - 0 1 5}$ \\
\hline Rastrigin & $1.1182 \mathrm{E}+02$ & $1.05 \mathrm{E}+05$ & $1.31556 \mathrm{E}+002$ & $\mathbf{1 . 1 3 6 0 2 E}+\mathbf{0 0}$ \\
\hline Ackley & $3.335 \mathrm{E}+002$ & $6.4257 \mathrm{E}+02$ & $8.74222 \mathrm{E}+00$ & $\mathbf{4 . 9 4 6 5 1 4 E}-10$ \\
\hline
\end{tabular}

Table VI Comparison of ABC, L-ABC with variants of PSO and DE over shifted benchmark functions

\begin{tabular}{|l|l|l|l|l|}
\hline \multicolumn{1}{|c|}{ Function } & DEwSAcc & EPUS-PSO & ABC & $\underline{\text { L-ABC }}$ \\
\hline Shifted Sphere & $5.5194 \mathrm{E}-11$ & $6.91 \mathrm{E}+001$ & $5.6776 \mathrm{E}-007$ & $\mathbf{6 . 8 1 5 7 E - 0 1 3}$ \\
\hline Shifted Rastrigin & $2.8699 \mathrm{E}+002$ & $3.27 \mathrm{E}+03$ & $7.73042 \mathrm{E}+01$ & $\mathbf{6 . 9 9 1 4 E}+\mathbf{0 0}$ \\
\hline Shifted Ackley & $\mathbf{7 . 0 9 7 3 E - 0 7}$ & $5.66 \mathrm{E}+00$ & $2.94500 \mathrm{E}-01$ & $6.5574 \mathrm{E}-006$ \\
\hline
\end{tabular}




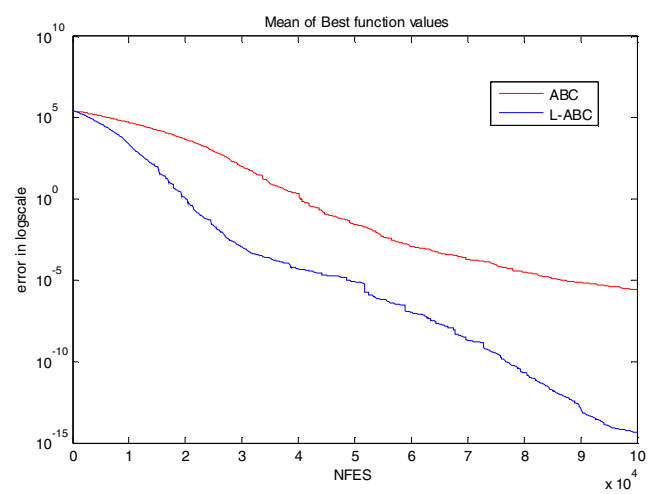

Fig 1 Convergence plot of Sphere Function

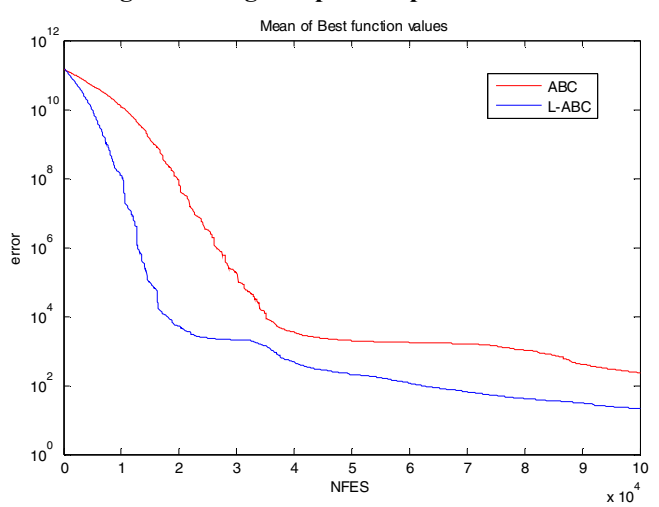

Fig 2 Convergence plot of Rosenbrock Function

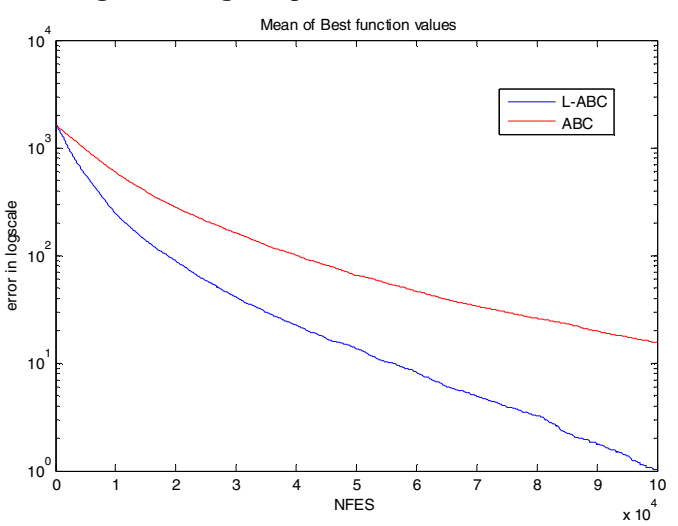

Fig 3 Convergence plot of Rastrigin Function

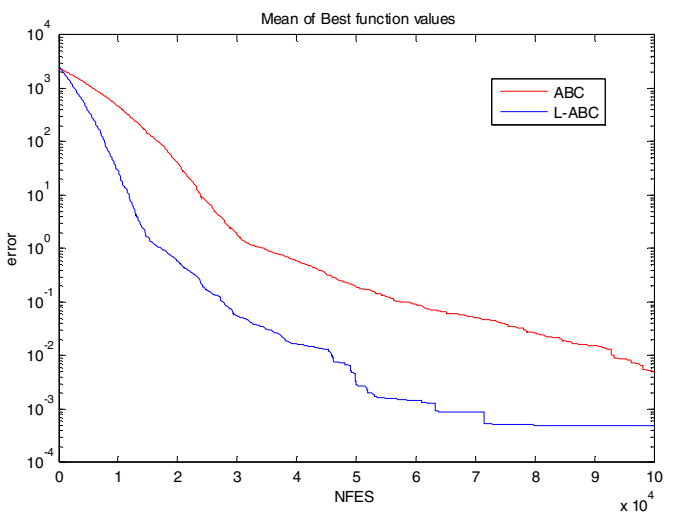

Fig 4 Convergence plot of Grienwank Function

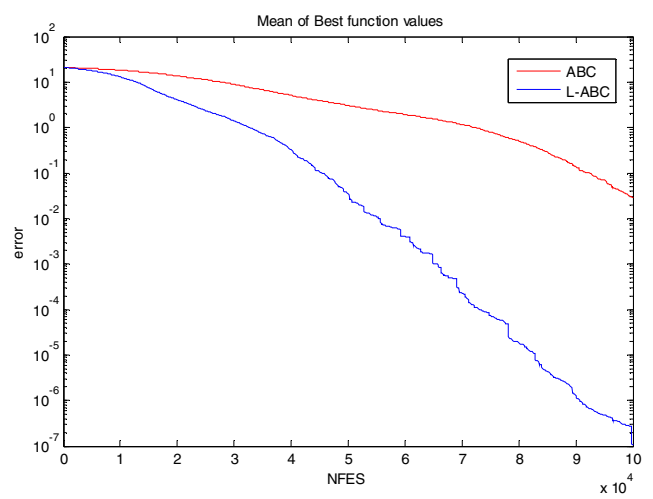

Fig 5 Convergence plot of Ackley Function

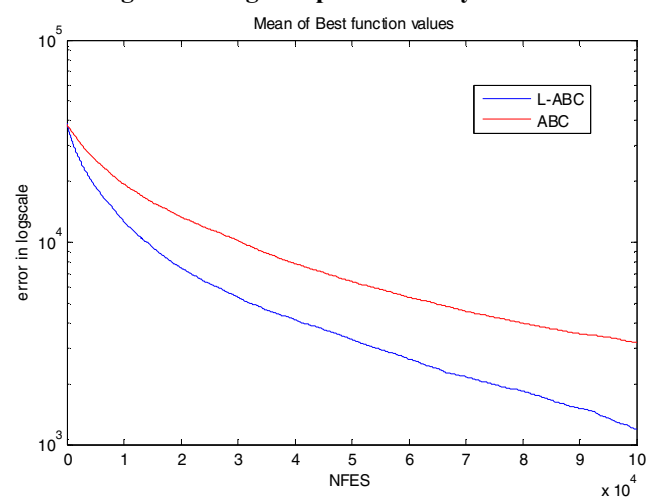

Fig 6 Convergence Plot of Schwefel Function

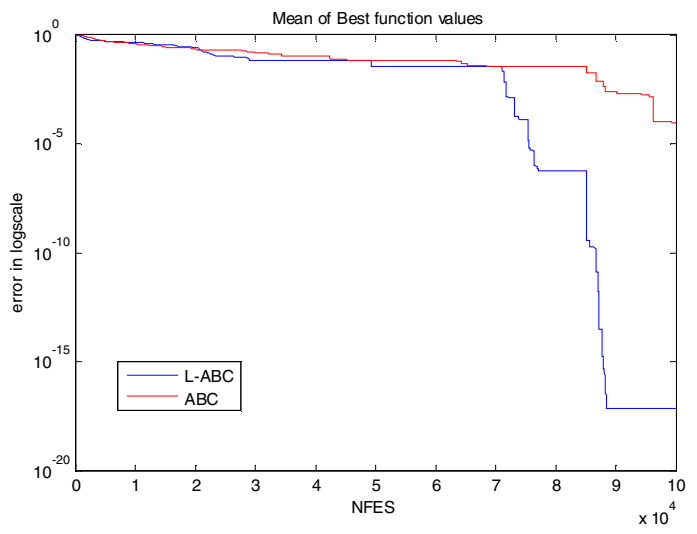

Fig 7 Convergence plot of Easom Problem

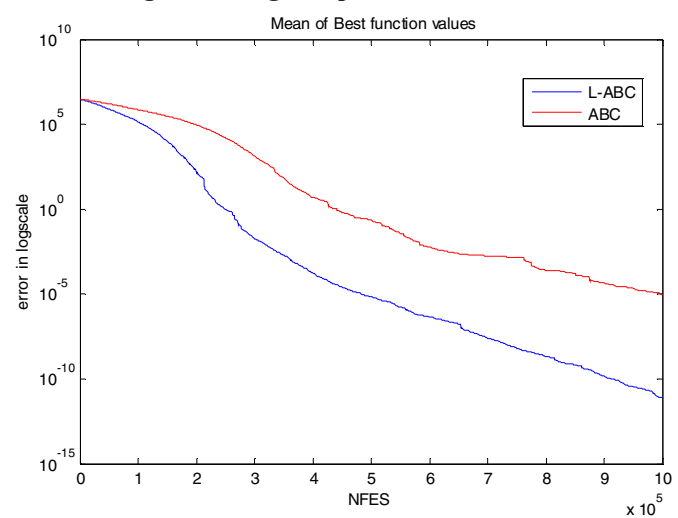

Fig 8 Convergence plot of Shifted Sphere Function 


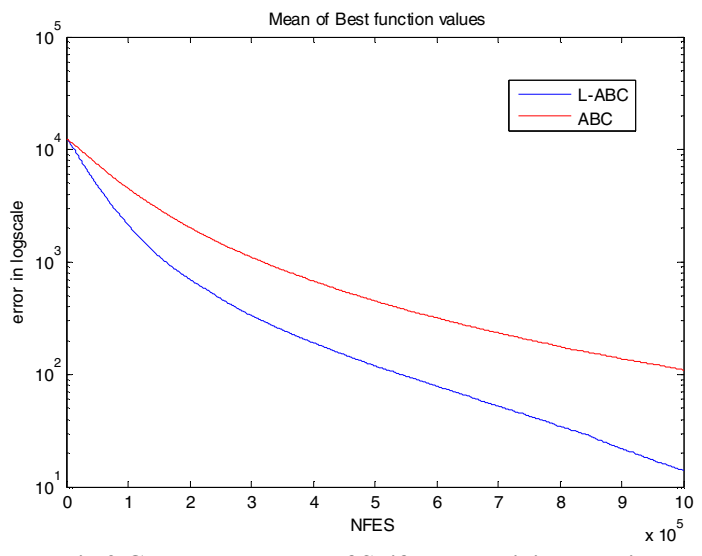

Fig 9 Convergence plot of Shifted Rastrigin Function

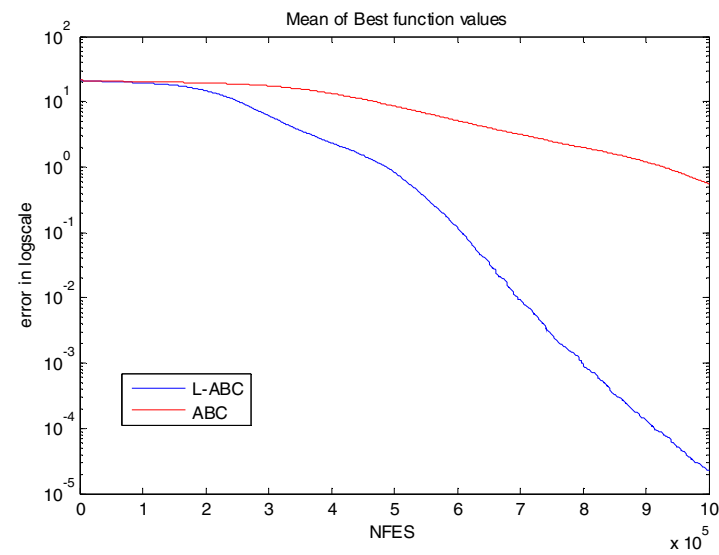

Fig 10 Convergence plot of Shifted Ackley Function

\section{APPLICATION TO PARAMETER ESTIMATION For FREQUENCY-MODUALTED (FM) SOUND WAVES}

Frequency-modulate (FM) sound synthesis plays a vital role in several modern musical applications. In this section the proposed $\mathrm{L}-\mathrm{ABC}$ is used to optimize the parameters of an FM synthesizer. Genetic algorithms are being used for the FM synthesizer in past [17 18]. Here, the system is designed in such a way that it can automatically generate sounds similar to the target sounds. The target sound is a .wav file. The L-ABC initializes a set of parameters, and the FM synthesizer generates the corresponding sounds. In the feature extraction step, the dissimilarities of features between the target sound and synthesized sounds are used to compute the fitness value. This process repeats until the synthesized sounds become very similar to the target.

The problem involves determination of six real parameters $\vec{X}=\left\{a_{1}, \omega_{1}, a_{2}, \omega_{2}, a_{3} \omega_{3}\right\}$ of the FM sound wave given by (6) for approximating it to the sound wave given in (7) where $\theta=2 \pi / 100$. The parameters are defined in the range $[-6.4,+6.35]$

$y(t)=a_{1} \cdot \sin \left(\omega_{1} \cdot t \cdot \theta+a_{2} \cdot \sin \left(\omega_{2} \cdot t \cdot \theta+a_{3} \cdot \sin \left(\omega_{3} \cdot t \cdot \theta\right)\right)\right)$ $y_{0}(t)=1.0 \sin (5 . t . \theta-1.5 \sin (4.8 t \cdot \theta+2 . \sin (4.9 . t . \theta)))$

The objective is to minimize the sum of square errors given by (8). This problem is a highly complex multimodal function having strong epistasis (interrelation among the variables), with the optimum value of 0.0 .

$$
f(\vec{X})=\sum_{t=0}^{100}\left(y(t)-y_{0}(t)\right)^{2}
$$

Figure 13 shows the convergence of the Frequency Modulate sound synthesis function. The L-ABC had outperformed the $\mathrm{ABC}$ because of its improved mutation strategy. To perform this application we had considered the algorithmic parameters that of Table II and the population of artificial bee was set to 20. A termination criterion of 1,00,000 Functional Evaluations was used for this problem.

Table VII. Fitness values obtained by ABC and L-ABC

\begin{tabular}{|l|l|l|}
\hline & $\underline{\mathbf{A B C}}$ & $\underline{\mathbf{L}-\mathbf{A B C}}$ \\
\hline$\underline{\text { Fitness }}$ & 2.0456 & $5.7595 \mathrm{e}-005$ \\
\hline
\end{tabular}

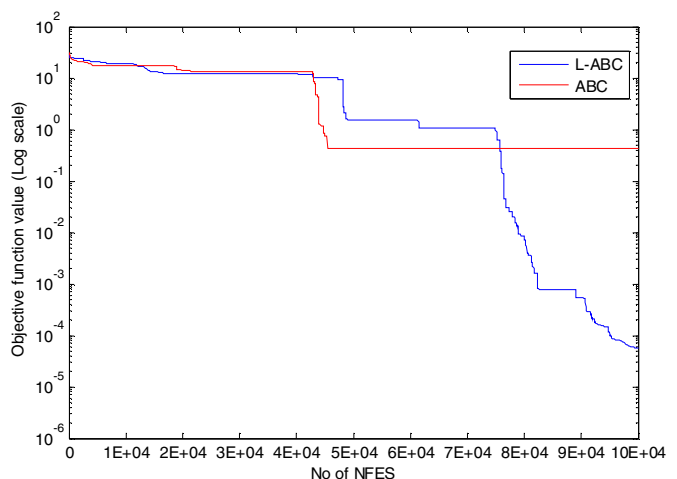

Fig 11 Progress towards the optimum solution for the frequency modulator synthesis Problem

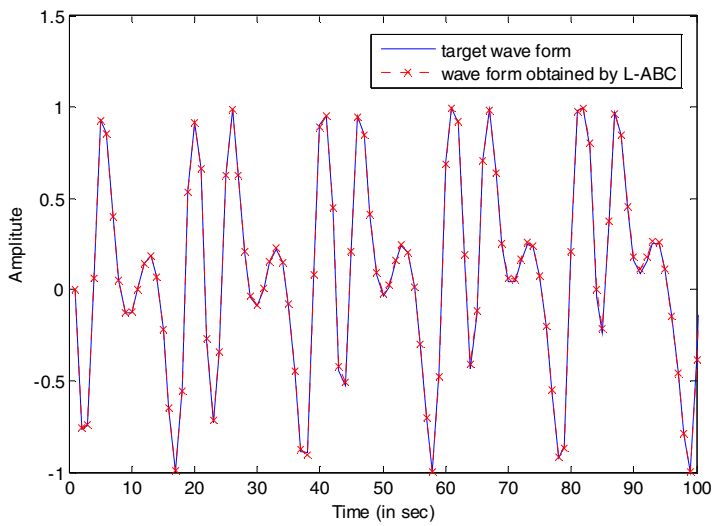

Fig 12 Actual target sound and the waveform synthesized by L-ABC

Table VII indicate the superior performance of L-ABC over basic $\mathrm{ABC}$ in terms of final accuracy and convergence speed from Fig. 11. Also Fig 12 shows that the waveform 
estimated by L-ABC achieves a high level of correspondence with the actual FM sound wave

\section{CONCLUSIONS}

Artificial Bee Colony Algorithm with Levy mutation was formulated and its performance assessment was given for the 7-high dimensional test functions and 3-high dimensional shifted test functions proposed for the special session of Large Scale Global Optimization at 2008 IEEE World Congress on Computational Intelligence. The presence of Levy probability distribution in the basic structure of $\mathrm{ABC}$ increases its efficiency without imposing much burden on its rate of convergence. L-ABC showed better results than its classical counterpart for all of the tested problems. Further, the mutation scheme outperformed a state of the art variant of PSO, GA and also variants of DE in solving high dimensional functions.

Future research may focus on extending L-ABC for solving constrained optimization problems and modifying it suitably for multiobjective optimization problems. Also instead of using Rechenberg's rule of mutation, we can apply it adaptively.

\section{ACKNOWLEDGEMENTS}

This work was supported by the Czech Science Foundation, under the grant no. GA102/09/1494.

\section{REFERENCES}

[1] D. Karaboga, B. Basturk, A powerful and efficient Algorithm for Numerical Optimization: Artificial bee Colony (ABC) Algorithm, Journal of Global Optimization, Volume: 39, Issue:3, pp: 459-172, 2007.

[2] D.Karaboga, B.Basturk, On The Pefrormance of Artificial bee Colony (ABC) Algorithm, Applied Soft Computing, Volume 8, Issue 1, pp: 687-697, 2008.

[3] D. Karaboga, C. Ozturk, A Novel Clustering Approach: Artifcial bee Colony (ABC) Algorithm, Applied Soft Computing, Volume 11, Issue 1, pp: 652-657, 2011

[4] N. Karaboga, A new design method based on artificial bee colony algorithm for digital IIR filters, Journal of the Franklin Institute, Volume 346, Issue 4, pp: 328-348

[5] Samrat Sabat, Siba K Udgata and Ajith Abraham, Artificial Bee Colony Algorithm for Small Signal Model Parameter Extraction of MESFET, Engineering Applications of Artificial Intelligence, Elsevier Science, Netherlands, 2010.

[6] G. Zhu, S. Kwong, Gbest-guided artificial bee colony algorithm for numerical function optimization, Applied Mathematics and Computation. Volume 217, Issue 7, pp: 3166-3173, 2010

[7] XS. Yang, Engineering Optimizations via Nature-Inspired Vitrual Bee Algorithms, In: Lecture notes in computer science, Springer (GHMB), pp: 317-323, 2005.

[8] K. Sundareswaran, VT. Sreedevi, Development of novel optimization procedure based on honey bee foraging behaviour. In: IEEE International Conference on systems, man and cybernetics, 2008

[9] Bahriye Akay, Dervis Karaboga, A modified Artificial Bee Colony algorithm for real-parameter optimization, Information Sciences, In Press, Corrected Proof, Available online 27 July 2010, ISSN 00200255, DOI: 10.1016/j.ins.2010.07.015

[10] Ajith Abraham, Ravi Kumar Jatoth and A. Rajasekhar, Hybrid Differential Artificial Bee Colony Algorithm, Journal of Computational and Theoretical Nanoscience, USA, 2011.
[11] Chang-Yong Lee; Xin Yao;, "Evolutionary programming using mutations based on the Levy probability distritbuion, "Evolutionary Computation, IEEE Trans on. Vol. 8, no. 1, pp. 1-13, Feb 2004.

[12] K. Tang, X. Yao, P. N. Suganthan, C. MacNish, Y.P. Chen, C.M. Chen, and Z. Yang, "Benchmark Functions for the CEC'2008 Special Session and Competion on Large Scale Global Optimization, " Technical Report, Nature Inspired Computation and Applications Laboratory, USTC, China, http://nical.ustc.edu.cn/cec08ss.php, November 2007.

[13] P. Levy, Theorie de l'Addition des Veriables, Aleatories, Paris, France: Gauthier-Villars.

[14] G. Crina, A. Ajith, and H. Aboul, "A line search approach for high dimensional function optimization,". Telecommunication Systems, ISSN: 1018-4864, Vol. 46, Springer Netherlands

[15] Zamuda, A.; Brest, j; Boskovic, B.; Zummber, V.:, "Large Scale Global Optimization using Differential Evolution with self-adaption and cooperative co-evolution, " Evolutionary Computation, 2008 CEC'2008. (IEEE World Congress on Computational Intillegence). IEEE Congress on, vol. no. pp. 3718-3725, June 2008.

[16] Sheng-Ta Hsieh; Tsung-Ying sun; Chan-Cheng Liu, Shang-Jeng Tsai;, "Solving large scale global optimization using improved Particle Swarm Optimizer, "Evolutionary Computation, 2008. CEC 2008. Pp. 1777-1784, June 2008.

[17] A. Horner, J. Beauchamp, and L. Haken. "Genetic algorithms and their application to FM matching synthesis," Comput. Music, J., vol. 17, pp. 17-29, 1993.

[18] F. Herrera and M. Lozano, "Gradual distributed real-coded genetic algorithm, " IEEE Trans Evol Comput. Vol. 4, no. 1, pp. 43-62, April 2000 . 